Auflage des Buches Raum, Zeit, Materie (siehe die Besprechung in diesem Band der Monatshefte). Wir können dem Verfasser dafür dankbar sein, daß er seine interessanten spanischen Vorlesungen auch der deutschen Leserwelt zugänglich gemacht hat.

J. Lense.

\title{
Sur l'homéomorphie de deux figures et de leurs voisinages.
} Diss. Straßburg 1921. Von L. Antoine.

Zwei homöomorphe, das heißt aufeinander umkehrbar eindeutig und umkehrbar stetig abbildbare Figuren können diese Homöomorphie in drei verschiedenen Graden aufweisen.

1. Sie werden durch eine topologische Abbildung der ganzen Räume, in denen sie definiert sind, ineinander übergeführt.

2. Sie werden durch eine topologische Abbildung irgend zweier sie umgebenden Mengen ineinander iibergeftibrt ohne daß 1. zutrifft.

3 . Sie sind topologische Bilder voneinander, ohne daß 1: oder 2, zutrifft.

Der Autor untersucht nun in gewissen besonders wichtigen Sonderfällen, welcher der drei Grade vorliegt.

\section{Jordansche Kurven.}

Für irgend zwei Jordanbögen ohne mehrfache Punkte (in Räumen gleicher Dimensionen) liegt 1. vor, ebenso für geschlossene Jordankurven ohne mehrfache Punkte in zwei Ebenen. Dagegen zeigen Beispiele, daß bei Jordanbögen und geschlossenen. Jordankurven 2. und 3, auftreten können, wenn sie mehrfache Punkte aufweisen oder in Räumen von mehr als zwei Dimensionen liegen.

Dabei ist das Resultat über zwei ebene Jordankurven ohne "mehrfachen Punkt ohne Benutzung des Jordanschen Kurvensatzes gewonnen. Dieser kann also hier aus der Tatsache, daß er für den Kreis gilt, allgemein gefolgert werden.

II. Die perfekten zusammenhanglosen geschränkten Mengen.

Zusammenhanglos (partout discontinu) heißt dabei eine Menge, welche zwischen je zweien ihrer Punkte nicht zusammenhängend ist.

Hier wird gezeigt:

Jede geschränkte abgeschlossene zusammenhanglose Menge liegt auf einem Jordanbogen ohne mehrfachen Punkt (Denjoy).

Irgend zwei geschränirte perfekte zusammenhanglose Mengen sind homöomorph (Denjoy).

Für sie liegt 1. vor, wenn sie in zwei Ebenen liegen. In Räumen höherer Dimensionen können auch 2. und 3. auftreten. Besonders interessant ist hier eine Menge P, welche trotz ihrer Zusammenhanglosigkeit doch von jeder einfach geschlossenen Fläche, welche irgend zwei ihrer Punkte trennt, geschnitten wird, und zwar in nicht abzählbar vielen Punkten.

Es hieße dem Autor unrecht tun, wollte man in diesen Resultaten seine ganze Leistung sehen. Mir wenigstens ist die Durchführung im einzelnen, so zum Beispiel die Hersteling der Abbildung zwischen ebenen Jordankurven mit Verwendung der De la Vallée Poussinschen schainons\&, eine Reihe von Hilfssätzen und die als Hilfsmittel zur Konstrulktion der Beispiele getriebene Topologie des Torus das Interessantere. Freilich glaube ich auch, daß die hier behandelten Dinge einer einfacheren Darstellung fähig sein werden, sobald die allgemeine Topologie dazu die Ausdrucksmittel geliefert haben wird.

L. Vietoris.

Analytische Geometrie des Raumés. Von G. Salmon und W. Fiedler unter Mitwirkung von A. Brill, neu herausgegeben von K. Kommerell. I. Teil. Die Elemente und die Theorie der Flächen zweiter Ordnung. 5. Auflage. XIV und 612 Seiten. B. G. Teubner, Leipzig und Berlin 1922.

Das altbekannte und berïhmte Lehrbuch der analytischen Geometrie des Raumes von Salmon-Fiedler erfuhr in dieser 5. Auflage eine vollständige und durchgreifende Umarbeitung, die, aufs wämste zu begriifen ist, weil in ihr die von

Monatsh, für Mathematik u. Physik. XXXIrI. Band. 